\title{
AVALIAÇÃO DE SIMULAÇÃO EMPRESARIAL: UM ESTUDO NA UNIVERSIDADE AUTÔNOMA DE LISBOA
}

\author{
ENTERPRISE SIMULATION EVALUATION: \\ A STUDY IN THE AUTONOMOUS UNIVERSITY OF LISBON
}

Recebido em: 27/11/2012 Aprovado em: 12/03/2013
Avaliado pelo sistema double blind review
Editora Científica: Manolita Correia Lima

JANAINA PIANA janainapiana@gmail.com

FUNDAÇÃO GETÚLIO VARGAS - ESCOLA BRASILEIRA DE ADMINISTRAÇÃO PÚBLICA E DE EMPRESAS

ROLF HERMANN ERDMANN

UNIVERSIDADE FEDERAL DE SANTA CATARINA

\section{RESUMO}

O interesse de pesquisadores em verificar os processos de ensino-aprendizagem, de forma a contribuir para a prática da gestão, educação e desenvolvimento gerencial, tem aumentado consideravelmente (TAPsCOTT, 1997). Neste panorama, buscando potencializar o ensino da Administração, os autores deste trabalho desenvolveram um Jogo Empresarial. Buscou-se neste artigo, portanto, avaliar o Jogo e o método de aplicação com base em três dimensões: (I) funcionamento operacional do Jogo, (2) a técnica de aprendizagem de Jogos de Empresas, e (3) a aprendizagem do aluno. Para tal, utilizou-se o método de quase-experimento, com aplicação de testes antes e depois, bem como utilizou-se da aplicação de questionários aos alunos participantes. A aplicação do Jogo ocorreu em duas turmas de graduação em Administração da Universidade Autônoma de Lisboa (UTL). Os resultados mostraram que a participação no jogo levou ao incremento da base teórica dos alunos. Porém, vencer não é fator relevante se correlacionado à aprendizagem. Ainda, o método apresentou-se motivador e estimulante, porém o funcionamento do jogo mostrou algumas fragilidades. Ao final, sugestões foram apontadas, relacionadas a interface do modelo, visando o aperfeiçoamento do Jogo a fim de consolidar uma ferramenta que contribua no processo de ensino-aprendizagem em Administração.

Palavras-chaves: jogos de empresa; simulação; ensino-aprendizagem.

\section{ABSTRACT}

Researchers' in reviewing teaching and learning processes in order to contribute to the practice of management education and management development has increased considerably (TAPSCOTT, 1997). So, seeking to enrich the Business Administration education, the authors of this study developed a Business Game. This paper therefore aims to evaluate the game and its method of application based on three aspects: (1) the operational functioning of the Game, (2) business games learning technique, and (3) student learning. The quasi experiment method, with the application of tests before and after, was employed, as were questionnaires, for the participating students. The Business Game was applied to two undergraduate Business Administration classes at the Autonomous University of Lisbon. The results showed that participation in the game led to an increase of the students' theoretical foundations. Winning, however, is not a relevant factor when correlated to learning. Furthermore, the method proved to be motivating and stimulating, but the functioning of the game presented some weaknesses. Finally, suggestions were pointed out, related to the interface model, aimed at improving the game in order to build a tool that helps in the teaching and learning of Business Administration.

Keywords: business games; simulations; teaching and learning. 


\section{INTRODUÇÃO}

Os profissionais da Administração tomam suas decisões diante de inúmeras circunstâncias e problemas utilizando suas experiências, seus conhecimentos, suas habilidades e valores. Diante disso, os gestores têm buscado formas mais estruturadas e objetivas para entender e lidar com a imprevisibilidade e a incerteza da tomada de decisão, como forma de garantir a competitividade organizacional.

Neste contexto os cursos de Administração precisam focar na formação de gestores qualificados e, principalmente, no desenvolvimento do aprendizado sistêmico das diversas áreas da Administração como forma de auxiliar no desempenho profissional.

Entretanto, o que se percebe é que as escolas de Administração possuem dificuldades para atender a necessidade de oferecer uma qualificação significativa aos estudantes. A dificuldade pode estar na falta de uma integração adequada entre a universidade, as empresas e o mercado, isso se dá pelo distanciamento entre as competências exigidas pelo mercado e o que vem sendo passado aos discentes pelas instituições de ensino em Administração. Andrade et al. (2004) em sua pesquisa concluem haver um gap entre a formação dada pelos cursos de Administração e as expectativas do mercado de trabalho.

Desta forma, os cursos de Administração e/ou Gestão tradicionais passaram a sofrer inúmeras críticas, fazendo crescer o interesse no processo de aprendizagem. Isso fez com que a aprendizagem se consolidasse como uma área de pesquisa e prática no campo das organizações. Cabe ressaltar que, no ensino as atividades estão centradas no professor e todo o seu repertório de conhecimento e competências; já, na aprendizagem, as atividades estão concentradas no aluno, com suas capacidades e possibilidades de desenvolvimento (OliveIra; SAUAia, 20II).

Carniel (2002), Gerber (2000), Silva (1998) e Faria (1997) mencionam que o uso dos Jogos Empresariais juntamente com o ensino tradicional vem potencializando a melhoria da qualidade do processo de aprendizagem e ensino, principalmente em cursos de Administração, por meio da 
exploração da complementaridade que estas formas propiciam entre elas. Portanto, os jogos de empresas surgem como uma ferramenta de aprendizagem que integra a teoria à prática por oportunizar ao aluno vivenciar os conceitos aprendidos em sala de aula o mais próximo do real (MOscovici, 1985). A utilização deste recurso didático na formação de gestores pode promover a preparação de profissionais mais completos e maduros para enfrentar o mercado de trabalho. Isso recai, principalmente, em disciplinas como Administração da Produção, que segundo Silva (20I2) está desgastada por práticas de ensino-aprendizagem de exagerado tecnicismo. Em outras palavras, os alunos dos cursos de Administração em geral não são confrontados com o conceito de produção. Formam um estereótipo que associa a administração da produção ao tecnicismo inerente ao ato de produzir, afastando-a de seu escopo de competências. Esquecem, no entanto, do papel da gestão, o que leva a que isto seja relegado a outros profissionais, especialmente aqueles com formação tecnológica na área dos produtos e processos gerenciados. A gestão ou a administração é assunto da Administração. Daí pretender-se com esta iniciativa ajudar a fixar o administrador em uma das grandes áreas da Administração.

Assim, o problema de pesquisa concentra-se na verificação das contribuições e/ou limitações da utilização de um Jogo Empresarial específico, criado pelos autores, para o processo de aprendizagem de alunos de Administração. Para tal, buscar-se-á avaliar o funcionamento do novo Jogo, na perpectiva do aluno; avaliar o comportamento e aprendizagem dos alunos; e, avaliar a técnica de aprendizagem. 


\section{JOGOS EMPRESARIAS}

Simulação de Gestão Empresarial, Jogo de Gestão, Jogo de Empresas, Jogo Empresarial, Jogo de Negócios ou Business Games se apresentam como uma ferramenta cujo objetivo é levar o aprendiz a vivenciar situações semelhantes a situações reais, e que permite a avaliação por meio da análise das possíveis consequências decorrentes de decisões adotadas e que por isso tem desempenhado um papel importante como instrumento didático. Desenvolvido por meio de um modelo matemático que determina as características técnicas, organizacionais e administrativas das empresas e as relações destas com o meio, o jogo favorece o desbloqueio psicológico que possa existir no aprendiz, além de desenvolver habilidades como: abstrair, organizar e usar informações, prever e planejar, combinar papéis de generalista e especialista, auxiliar no trabalho em grupo, processar informações importantes e experimentar novas ideias (MARTINELLI, I987; SAUAIA, I995; TANABE, 1977).

Na simulação os problemas são interligados. A execução da tomada de decisão passa a ser global. Assim, não basta tomar boas decisões isoladamente, senão tomar uma decisão que leve em consideração todas as restrições de cada área simulada. Isto torna a simulação um método pedagógico completo para o crescimento e integração dos conhecimentos, onde não se lida com imagens fixas, mas sim como dinâmicas complexas. Do ponto de vista da aprendizagem, a simulação introduz a vocação indutiva. $\mathrm{Na}$ indução as conclusões são tiradas com base em de casos particulares. No caso, num processo de simulação, baseada em uma experiência singular que o aluno participa, ele tende a chegar a conclusões mais gerais a respeito do assunto que está sendo simulado (HOUE; GUIMARÃEs; CHERNOV, 2000).

As vantagens à adesão aos jogos empresariais são: maior compreensão dos conceitos antes considerados abstratos; conscientização da necessidade de um realinhamento de atitude e de comportamento no atual momento de mudanças; maior possibilidade de comprometimento do grupo com resultados; reconhecimento do próprio potencial e das dificuldades individuais; resgate do lúdico - essência do ser humano; resgate do potencial 
criativo e descoberta de possibilidades não consideradas anteriormente; possibilidade de mensuração de resultados durante os jogos simulados, possibilitando avaliações comparativas com a realidade empresarial; maiores chances de desenvolvimento de habilidades técnicas, conceituais e interpessoais (GRAMIGNA, 1995).

Neste sentido, Martinelli (1987) corrobora salientado que os jogos apresentam-se como uma ferramenta de aprendizagem vivencial cujo objetivo é levar o aprendiz a vivenciar situações semelhantes a situações reais e por isso tem desempenhado um papel importante nos últimos tempos como instrumento didático. Essa vivência favorece o desbloqueio psicológico que possa existir no aprendiz, além de desenvolver habilidades como: abstrair, organizar e usar informações, prever e planejar, combinar papéis de generalista e especialista, auxiliar no trabalho em grupo, processar informações importantes e experimentar novas ideias. Ainda, Bernard (2006) afirma que com os jogos empresariais, os estudantes têm a possibilidade de praticar, por meio da simulação, os conceitos teóricos adquiridos nas diversas disciplinas de sua formação acadêmica.

Assim, o método de jogos de empresa possibilita a integração das disciplinas do curso, a transferência dos conceitos teóricos para o campo prático (BERNARD, 2006) e também, por trazer em si uma abordagem vivencial, possibilita uma reflexão sobre a tomada de decisão nas organizações.

Estudos apontam que a integração da prática com a teoria dentro do curso é a principal vantagem da simulação gerencial (BERNARD, 2006). Dentre os autores brasileiros que mostravam essa vantagem citam-se Protil (2005) e Sauaia (1995). Com o uso da simulação gerencial, então, é possível melhorar a interação entre prática e resultado, que é um dos pontos apontados como preocupantes no ensino de Administração.

Segundo Bernard (2006) a simulação centraliza a aprendizagem nos problemas apresentados ao estudante que analisa as possibilidades que podem ser adotadas e decide qual utilizar para resolução do problema em foco, responsabilizando-se em grande parte pelo seu próprio ensino. A simulação traz situações do cotidiano de uma organização e/ou do 
mercado em que se está inserida, desta forma os estudantes, com base em informações que lhes são repassadas, tomam decisões em condições próximas as reais.

Portanto, a utilização da simulação gerencial traz a vantagem do envolvimento das pessoas em uma atividade na qual, além de vivenciá-la, elas têm a oportunidade de analisar o processo de forma crítica, extrair algum insight útil desta análise e aplicar o aprendizado em seu cotidiano (GRAMIGNA, 1995).

Como exemplos de jogos de empresas, especificamente na área de Administração da Produção, Badurdeen et al. (2010) em sua pesquisa fizeram um mapeamento dos jogos empresariais relacionados à área. Segundo os autores, embora, certamente existam simuladores específicos de Administração da Produção que não sejam disponíveis para avaliação, o conjunto apresentado no Quadro I pode ser uma representação razoável dos jogos empresariais orientados para Administração da Produção.

Quadro I Jogos de Empresas em Administração da Produção

\begin{tabular}{|lll|}
\hline Nomenclatura do Jogo & Ano & Criadores (fonte publicada) \\
\hline Beer Game & 1963 & MIT (Jarmain, 1963) \\
\hline Uk Paper Clip Simulation & 1994 & Universidade de Kentucky (Hall, 1994) \\
\hline Uk Circuit Board Simulation & 1994 & Universidade de Kentucky (Lean training, 1994) \\
\hline Buckingham Lean Game & 1995 & Bicheno (1995) \\
\hline Circuit Board Simulation & 1998 & NIST-MEP (NIST/MEP, 1998) \\
\hline Cylinder Factory Simulation & 2003 & $\begin{array}{l}\text { Universidade de Kentucky (Badurdeen } \\
\text { et al., 2008; Price, 2008) }\end{array}$ \\
\hline Timewise Simulation & 2003 & MEP (Verma, 2003) \\
\hline Pipe Factory Simulation & 2003 & Universidade de Dayton (Verma, 2003) \\
\hline Lean Sim Machine & 2003 & Donnelly Corporation (Verma, 2003) \\
\hline Lean Enterprise Value Simulation & 2003 & Lean Aerospace Initiative (McManus \\
\hline Box Game Simulation & & et al., 2007) \\
\hline Dice/Parade Game & 2003 & WCM Associates (Verma, 2003) \\
\hline Lean Product Development Simulation & 2003 & Universidade de Tennessee (Verma, 2003) \\
\hline Cellular Manufacturing Simulation & 2003 & Visionary Products Inc. (Verma, 2003) \\
\hline Widget Factory Simulation & 2003 & Lockwood Greene (Verma, 2003) \\
\hline
\end{tabular}




\begin{tabular}{|c|c|c|}
\hline Nomenclatura do Jogo & Ano & Criadores (fonte publicada) \\
\hline Ball Bearing Factory & 2003 & Gary Randall (Verma, 2003) \\
\hline Nist Buzz Electrionics Simulation & 2003 & $\begin{array}{l}\text { Illinois Manufacturing Extension } \\
\text { Center (Stier, 2003) }\end{array}$ \\
\hline 5s Simulation & 2003 & NIST-MEP (Verma, 2003) \\
\hline Setup Reduction Simulation & 2003 & NIST-MEP (Verma, 2003) \\
\hline Paper Airplane Game & 2003 & Northrop Grumman (Verma, 2003) \\
\hline 5s Mini-Factory Simulation & 2004 & $\begin{array}{l}\text { Universidade de Kentucky (Lean Boot } \\
\text { Camp, 2004) }\end{array}$ \\
\hline Wagons-R-Us Simulation & 2004 & Blust and Bates (2004) \\
\hline Paper Airplane Exercise & 2004 & Billington (2004) \\
\hline Furniture Factory Simulation & 2004 & $\begin{array}{l}\text { University of Kentucky (Boot camp II Course, } \\
\text { 2004a; Badurdeen et al., 2008) }\end{array}$ \\
\hline Veebot Simulation & 2004 & University of Kentucky (Lean Certification, 2004) \\
\hline Ship Repair Design Process Simulation & 2005 & $\begin{array}{l}\text { Old Dominion University (Verma, 2007; Verma } \\
\text { et al., 2005) }\end{array}$ \\
\hline Ship Repair Supply Chain Simulation & 2005 & $\begin{array}{l}\text { Old Dominion University (Verma, 2007; Verma } \\
\text { et al., 2005) }\end{array}$ \\
\hline Ship Repair Value Stream Mapping & 2005 & $\begin{array}{l}\text { Old Dominion University (Verma, 2007; Verma } \\
\text { et al., 2005) }\end{array}$ \\
\hline Ship Repair Simulation & 2005 & $\begin{array}{l}\text { Old Dominion University (Verma, 2007; Verma } \\
\text { et al., 2005) }\end{array}$ \\
\hline Ship Repair Scheduling Simulation & 2005 & $\begin{array}{l}\text { Old Dominion University (Verma, 2007; Verma } \\
\text { et al., 2005) }\end{array}$ \\
\hline Value Stream Mapping Board Game & 2005 & Verma and Devulapalli (2006), Verma (2007) \\
\hline Block Tower & 2005 & Verma (2007) \\
\hline Wagons-R-Us Simulation & 2006 & Winarchick and Carlisle (2006) \\
\hline Lean Enterprise Product Development & 2006 & $\begin{array}{l}\text { Lean Aerospace Initiative (McManus \& } \\
\text { Rebentisch, 2006a, 2006b) }\end{array}$ \\
\hline Lean Lego Simulation & 2006 & Fang, Cook, and Hauser (2007) \\
\hline Lean Lemonade Tycoon & 2007 & Ncube (2007) \\
\hline Lampshade Game & 2007 & Ozelkan and Galambosi $(2007,2008)$ \\
\hline Enterprise Simulation & 2007 & Universida de Kentucky (Maginnis, 2008) \\
\hline Nima'S Board Game & 2007 & MacMillian (2007) \\
\hline Engr. Design Class & 2007 & McManus et al. (2007) \\
\hline
\end{tabular}

Fonte: Adaptado de Badurdeen et al. (2010).

Contribuindo, Bouyer etal.(2005), emuma análise do ensino de Administração da Produção mostraram que os professores têm se preocupado muito com os 
métodos de ensino e com os recursos didáticos nessa área. Essa preocupação, segundo este autor, é justificada pelo fato da disciplina, encarada como sendo de foco predominantemente prático, estar hoje presa a condições metodológicas que a desviam em parte de seus objetivos.

Jarvis (1995) aponta que a abundância de recursos assegura o aprendizado, pois os alunos podem aprender respeitando seus próprios estilos pessoais. Além disso, cabe ressaltar que cada jogo ou recurso didático possui um objetivo e um propósito diferente.

O desenvolvimento da informática conduziu uma utilização generalizada de métodos de simulação e ferramentas em diversas áreas (JúNIOR; souZA; NASCIMENTO, 20I2), como por exemplo, ferramentas de apoio pedagógico. A utilização das novas tecnologias tem provocado diferentes impactos na educação. As descobertas digitais que permitem a interatividade introduzem novo comportamento na forma como as informações são absorvidas, tornando o indivíduo mais ativo no processo de aprendizagem. $\mathrm{O}$ uso da moderna tecnologia de informação desenvolve nos alunos a habilidade de obtenção de conhecimento com mais autonomia, tornando-os autodidatas e dificultando a aceitação dos métodos tradicionais de ensino. Daí o grande incremento ao uso de jogos empresariais (TAPSCOTT, 1997).

Consequentemente, vários estudos têm analisado a eficácia da utilização de jogos de empresas (SAUAIA, 2006; MITCHELL, 2004; JENSEN, 2003; SCHAFRANSKI, 2002; LOPES, 200I; MENDES, I997; SAUAIA, I995; TANABE, 1977). Segundo Seaton e Boyd (2008), há os acadêmicos que argumentam que as simulações são ferramentas pedagógicas extremamente úteis para o processo de aprendizagem, mas, há também, os acadêmicos que afirmam que as simulações seguirão para o declínio e desuso, sendo apenas uma moda passageira. Seaton e Boyd (2008) concluem que as simulações não mudarão os cursos empresariais, tão pouco são um método de aprendizagem perfeito, entretanto, podem ser uma parte muito importante do processo de aprendizagem. Gomes, Torrens e Cunha (2012), em seu estudo afirmam que a complexidade tecnológica influencia a resistência de professores para a utilização de computadores de forma geral, entretanto, este pode ser um dos motivos do não uso ou da descredibilidade quanto aos simuladores. 
Também, outros autores defendem que os jogos de empresas não podem ser tratados como ferramentas únicas de ensino. Devem ser integrados com outras técnicas de ensino como aulas expositivas, estudo de casos, seminários e outras técnicas, dentro do processo de ensino-aprendizagem, buscando atender o princípio de que nem todos os participantes possuem o mesmo aproveitamento perante as diversas maneiras de se transmitir conhecimento (SAUAIA, 2006; JENSEN, 2003; LOPES, 200I; MENDES, I997; SAUAIA, I995; TANABE, 1977).

Outros trabalhos acadêmicos no Brasil têm mostrado resultados auspiciosos para esta metodologia, todos concluindo pela excelência dela no processo de aprendizagem e treinamento das atividades de um administrador, de forma vivencial e mais motivadora, assim como uma preferência maior em relação a outras metodologias de ensino (BATISTA, 2004; HAZOFF, 2004; SCHAFRANSKI, 2002; SAUAIA, I995).

Entretanto, as pesquisas ainda são muito frágeis na tentativa de comprovar a validade dos jogos como uma prática educacional capaz de agregar conhecimento à formação do administrador, ou seja, de contribuir para uma aprendizagem significativa.

Perante o apresentado, uma lacuna é aberta para mais pesquisas que avaliem jogos empresariais e contribuam para um maior entendimento quanto à utilidade do método, em que casos o método se apresenta mais eficaz, e como a simulação é vista por alunos de outros países.

\section{AVALIAÇÃO EM JOGOS EMPRESARIAIS}

Buscando atender as novas e crescentes exigências na formação acadêmica, as instituições de ensino superior tem se preocupado com novas técnicas integrativas e construtivistas como formas de apreender conceitos, desenvolver habilidades e atitudes de forma integrada sem que haja a necessidade de criação de disciplinas específicas (ESCRIVÃo FILHO; RIBEIRO, 2007).

Baseando-se nessa percepção, às avaliações de aprendizagem se fundamentam nos princípios de que "educar é formar e aprender é construir o próprio saber" (HAIDT, I995, p. 286), não se reduzindo apenas a atribuir 
notas, deixando de avaliar apenas o produto final, e passando a avaliar todo o processo de ensino-aprendizagem.

Haidt (1995) apresenta os princípios básicos da avaliação. Para esses autores, o processo de avaliação está ligado ao processo de aprendizagem, sendo este planejado, pensado e realizado de forma coerente, ou seja, é um processo contínuo e sistemático, o que permite um contínuo reiniciar do processo de aprendizagem na busca dos objetivos finais, isto é, é também funcional.

Com relação às disciplinas de Jogos de Empresas, tradicionalmente, o método de avaliação mais utilizado, na graduação, é aquele baseado no desempenho das empresas fictícias. Esse tipo de abordagem parte do pressuposto de que as melhores empresas são aquelas onde se obteve um maior rendimento, o que, automaticamente, teria resultado em um maior aprendizado por parte dos alunos. Essa característica pode ser verdadeira em alguns casos, mas deve ser analisada a fim de compreender a dinâmica do processo de ensino-aprendizagem (STAHL; LOPES, 2004).

Diversas situações vivenciadas pelos autores dão conta de que quase sempre a performance da empresa não está relacionada com o nível de aprendizagem obtido, o que coloca em cheque esse pressuposto, largamente, aceito na área. Uma primeira questão é a interferência que fatores aleatórios, decorrentes de parâmetros definidos no cenário do jogo, podem causar nos resultados obtidos. Uma empresa pode ser considerada a vencedora da "competição" auxiliada por uma variável não controlável do ambiente, mesmo sem ter feito qualquer planejamento para o plano de decisões implementado. Da mesma forma, podem cenários regionais hostis, por exemplo, afetar negativamente o desenvolvimento da estratégia de uma empresa em beneficio das demais (STAHL; LOPES, 2004).

Na própria pedagogia atual é discutida a importância do erro no processo de aprendizagem (HOFFMAN, 1997), pois muitas vezes o aluno aprende mais nele do que nos momentos em que está acertando. O erro quando percebido pelo aluno, ou apontado pelo professor, tende a desencadear um processo de reflexão, levando o aluno a verificar porque errou e o que deveria ter feito. Refletindo sobre suas atividades e atitudes o aluno que errou consegue identificar novas situações e possibilidades que o aluno que "acertou" não irá 
descobrir, ou "aprender". O acerto não gera tal processo de reflexão, e deste modo não pode ser considerado que o aluno que errou não aprendeu o que o professor almejava com a atividade.

O que se busca realçar com isso é que um dos principais objetivos dos jogos de empresa é o processo de integração, a busca da visão sistêmica. A vantagem da utilização de jogos interativos neste processo se deve justamente à flexibilidade, uma vez que o feedback de informações é aleatório e imprevisível para os participantes, exigindo uma constante reavaliação das decisões, o que proporciona um grau ainda maior de motivação e aprendizado, principalmente porque esta atividade proporciona o processo contínuo de administração (planejamento, avaliação e controle) (STAHL; LOPES, 2004).

Stahl e Lopes (2004) propõem uma combinação de avaliações que reflitam os resultados alcançados segundo os objetivos pré-estabelecidos, não deixando de lado o desempenho das equipes, mas também não permitindo que apenas ele seja utilizado como referência para a avaliação da aprendizagem, conforme apresentado no Quadro 2.

Quadro 2 Estratégias de Avaliação para Simulações e Jogos de Empresas

\begin{tabular}{|ll|}
\hline Objetivos da avaliação & Tipo de Avaliação \\
\hline Conhecimentos Teóricos & Testes, provas dissertativas e planejamento estratégico \\
\hline Habilidades & $\begin{array}{l}\text { Relatórios periódicos, revisão do planejamento estratégico e } \\
\text { desempenho do grupo }\end{array}$ \\
\hline Comportamentos e atitudes & Auto-avaliação, avaliação inter-pares e fichas de observação \\
\hline
\end{tabular}

Fonte: Adaptado de Stahl e Lopes (2004).

Buscando avaliar os comportamento e atitudes, Motta, Melo e Paixão (20I2) por exemplo, analisaram a percepção dos alunos a respeito do significado dos jogos de empresas no processo de aprendizagem em Administração. Os autores coletaram os dados por meio de questões abertas e analisaram as respostas pelo método do Discurso do Sujeito Coletivo. Os autores identificaram que as principais contribuições do Jogo Empresarial foram: (a) o auxílio no treinamento para o processo decisório, encargo característico do administrador; (b) a vivência simulada das atividades empresariais; (c) o 
desenvolvimento de habilidades interpessoais para o trabalho em equipe; e (d) a aplicação dos conhecimentos adquiridos ao longo do curso de administração.

Para este estudo optou-se, para avaliar a aprendizagem quanto aos conhecimentos teóricos adquiridos pelos alunos, testes de caráter objetivo, do tipo verdadeiro/falso. Já para a avaliação das habilidades gerenciais desenvolvidas, estas foram associadas ao desempenho das equipes. Ainda, utilizou-se de um questionário de auto-avaliação para a verificação dos comportamentos e atitudes.

As mesmas ferramentas de avaliação foram utilizadas para verificação da adequação da técnica de ensino e do funcionamento do Jogo como um todo. 


\section{PROCEDIMENTOS METODOLÓGICOS}

A pesquisa, quanto aos fins, é caracterizada como explicativa, pelo fato da mesma se preocupar em identificar os fatores que contribuem ou determinam a ocorrência de um fato (ANDRADE, 2002). A maioria das pesquisas explicativas utiliza o método experimental, neste trabalho optouse pelo quase-experimento, na concepção antes e depois. A inviabilidade da distribuição aleatória e do controle, pelo fato do trabalho não ter objetivo de comparar a utilização de outro método de ensino, como, por exemplo, o ensino tradicional, sem o uso de ferramentas de simulação de laboratório, bem como pelo fato de inúmeras outras variáveis poderem influenciar tanto o grupo de controle quanto o grupo experimental, no caso de um experimento, justificou a utilização do quase-experimento.

A pesquisa foi realizada por meio da aplicação de um Jogo Empresarial criado pelos próprios autores, intitulado RCAP (Relações Complexas na Administração da Produção). O jogo, aqui testado, foi desenvolvido com o objetivo de auxiliar e estimular os alunos de Administração, à adquirir, esclarecere aperfeiçoar seus conhecimentos, além de desenvolver habilidades de gestão. O Jogo foi aplicado em duas turmas (diurno e noturno) ao nível de graduandos, estes formandos do curso de Administração, na disciplina de Estratégia Organizacional da Universidade Autônoma de Lisboa (UTL).

O Jogo RCAP simula um sistema de produção, com subsistemas, entendidos como áreas de um sistema de produção, que interagem com fatores de competitividade. Para construção do Jogo, portanto, mapeou-se as relações existentes criando-se uma modelagem matemática para cada relação de acordo com a situação da empresa em determinado momento. $\mathrm{O}$ aluno participante deveria fazer investimentos, com uma limitação de $€$ Ioo ০oo,০০, nas relações que considerasse mais relevante na situação da empresa apresentada. O resultado da performance da empresa se dá em valores por desempenho em custo, qualidade, confiabilidade, flexibilidade e rapidez.

Antes da aplicação do Jogo, primeiramente, foram expostos aos alunos os objetivos desta experiência que, especificamente, compreendem: 
- Avaliar o funcionamento do Jogo;

- Avaliar o comportamento e aprendizagem dos alunos;

- Avaliar a técnica de Jogos Empresariais.

Além disso, os alunos foram informados que o resultado atingido pela empresa seria considerado na avaliação do aproveitamento apenas de forma a beneficiá-los e em hipótese alguma poderia prejudicá- los, uma vez que os melhores colocados ganhariam pontos adicionais. Embora não possa prejudicá-los, os alunos foram informados sobre as vantagens pessoais que podem ser auferidas pela simples participação como jogador.

Quanto à aplicação do jogo, a mesma se deu conforme detalha Tanabe (1977) sobre um exercício de administração simulada:

A Iniciação do jogo (regras do jogo): ao iniciar-se o curso, a primeira atividade é fazer os alunos se familiarizarem com o meio ambiente simulado no qual irão, posteriormente, atuar. Eles se encontram nesse momento, na mesma situação de executivos que mudam de empresa e, uma vez instalado na nova, deve, antes de tomar decisões, aprender suas características. Para tanto, praticamente todos os jogos de empresas são providos de um "manual do participante" que contém as "regras do jogo", cuja estrutura permitirá aos alunos conhecer o contexto no qual terão que desenvolver suas atividades. Também podem ser estimulados a analisar os dados retrospectivos fornecidos e a descobrirem, por si mesmos, usando técnicas aprendidas em outros cursos, relações e parâmetros importantes para suas decisões. Desta forma, no primeiro dia de aula fez-se uma apresentação do Jogo, bem como, disponibilizou-se o Manual do Jogador. O Manual do Jogador trata-se de um documento que introduzirá o conceito do jogo, sua fundamentação teórica e explicará as regras de funcionamento do mesmo.

B Formação de equipes: após familiarizados com o ambiente simulado, foram formadas equipes de tamanho tão igual quanto possível, constituindo cada uma delas, uma empresa. Ainda, no primeiro dia de aula foram formadas as equipes que participariam do Jogo. Cada equipe constituiu-se de quatro integrantes. 
C As decisões dos jogos de empresas: cumpridas as etapas preparatórias, temse início o jogo. Constitui-se na repetição de tantos ciclos quantos o administrador do jogo julgar convenientes aos objetivos educacionais. Em cada sessão fornece-se a cada empresa um conjunto de relatórios que consubstanciam os resultados alcançados pela equipe em função das suas decisões e das de seus colegas, na aula anterior. Analisados os resultados anteriores, os alunos discutem as alternativas de ação e tomam as decisões pertinentes, visando aproveitar oportunidades surgidas, corrigir falhas e atingir o planejado. Após terem decidido o que fazer, registram suas decisões num formulário apropriado fornecido pelo administrador do jogo. Neste caso, houve apenas um ciclo de jogadas, onde as decisões foram tomadas extraclasse.

D Processamento das decisões: tanto o cálculo dos valores das variáveis determinados pelas decisões tomadas pelas equipes, como a apuração dos resultados, são feitos por meio das equações que compõem o modelo. Nesse momento, o uso de aparato tecnológico mostra-se como uma ferramenta que confere agilidade na alimentação e cálculo dos resultados, assim como precisão e confiabilidade dos dados gerados. Tais resultados são os relatórios a serem distribuídos às equipes e os novos dados históricos. A análise dos mesmos permitirá que as equipes descubram não só suas eventuais falhas, mas também, alguma coisa a mais a respeito do ambiente simulado em que estão atuando. Este ciclo, tomada de decisões, apuração dos resultados, análise dos mesmos, novas decisões, é repetido até o cumprimento dos objetivos didáticos do jogo. $\mathrm{O}$ administrador do jogo procura com o diálogo e a análise orientar as equipes no sentido de fazê-las reconhecer os instrumentos e técnicas da administração que as ajudariam em cada uma das situações que vão sucessivamente se configurando ao longo da simulação, incentivando-as, assim, a aplicar e testar os conhecimentos adquiridos durante o curso de Administração.

Durante o período dado para a tomada de decisões do Jogo, o facilitador buscou dialogar e orientar as equipes. Ao final, quando da entrega das decisões, os alunos receberam os resultados finais de suas empresas por 
meio de exposição oral, onde após por meio de questionário puderam dar a sua opinião sobre o Jogo, o método de aprendizagem e sobre seu próprio desempenho no Jogo. O resumo da aplicação e avaliação do jogo está apresentado no Quadro 3.

Para a coleta de dados foram utilizados os seguintes instrumentos:

Pré-teste e pós-teste - compostos de uma prova individual com de 20 questões versando sobre o conteúdo de Administração da Produção. Vale ressaltar que por serem alunos formandos, todos já haviam concluído a disciplina de Gestão de Operações, ou também conhecida Administração da Produção. Além disso, o pré-teste foi aplicado após aula expositiva sobre o conteúdo do pré-teste, bem como, foi disponibilizado um Manual com o conteúdo. O pré-teste tem por objetivo avaliar o conhecimento do aluno na área antes da participação no Jogo. E o pós-teste, procura identificar se houve uma evolução do conhecimento após a experiência.

Quadro 3 Aplicação do Jogo Empresarial

\begin{tabular}{|l|l|}
\hline Aula & Etapa 1 - Fase de Preparação \\
\hline $\begin{array}{l}\text { Primeira aula } \\
\text { (de } 50 \text { minutos) }\end{array}$ & \begin{tabular}{l} 
Apresentação e exposição a respeito do Jogo Empresarial a ser trabalhado. \\
Disponibilização e explicação do Manual do Jogo de Empresarial. \\
\hline Formação das equipes e aplicação do pré-teste.
\end{tabular} \\
\hline Extra-classe & Etapa 2 - Aplicação \\
\hline Extra-classe & $\begin{array}{l}\text { Primeira rodada do jogo (leitura de texto específico contido no manual forne- } \\
\text { cido, discussão em grupo e decisão) }\end{array}$ \\
\hline Aula & Etapa 3 - Finalização \\
\hline $\begin{array}{l}\text { Segunda aula, } \\
\text { de finalização } \\
\text { (50 minutos) }\end{array}$ & $\begin{array}{l}\text { Apresentação do desempenho no Jogo Empresarial } \\
\text { Aplicação de questionário aos participantes buscando a avaliação do jogo e a } \\
\text { própria avaliação do aluno quanto ao seu desempenho. Aplicação do pós-teste. }\end{array}$ \\
\hline
\end{tabular}

Para a avaliação dos resultados nos testes, foram comparadas as notas do pré e pós-teste das turmas, com o uso do teste estatístico " $t$ " de student, apropriado para comparar dois conjuntos de dados quantitativos, em termos de seus valores médios (BARBETTA, 2007). As hipóteses formuladas foram:

$\mathrm{H}_{\mathrm{o}}$ : a nota média dos alunos no teste não se altera com o uso do Jogo Empresarial. 
$\mathrm{H}_{\mathrm{r}:}$ a nota média dos alunos no teste aumenta com o uso do Jogo Empresarial.

\section{RESULTADO DO DESEMPENHO DAS EQUIPES NO JOGO}

Usando-se a média da variação da nota nos testes das equipes (x) e o desempenho das mesmas no Jogo (y) analisou-se a existência de correlação entre as duas variáveis por meio do coeficiente $r_{s}$ de Spearman. O uso do coeficiente $\mathrm{r}_{s}$ de Spearman se deu devido ao fato das variáveis de desempenho do Jogo assumirem valores muito discrepantes. Este coeficiente apresentouse mais apropriado, pois se utiliza da ordenação resultante da classificação obtida pelos jogadores e não de seus valores absolutos ou magnitudes (BARBETTA, 2007). As hipóteses estabelecidas foram:

$\mathrm{H}_{\mathrm{o}}$ : as variáveis x e y são não-correlacionadas;

$\mathrm{H}_{\mathrm{r}}$ as variáveis x e y são correlacionadas.

\section{QUESTIONÁRIO DE AVALIAÇÃO DO JOGO E DA PRÓPRIA} PARTICIPAÇÃO DO ALUNO COMO JOGADOR

O questionário foi desenvolvido recorrendo-se a diversos trabalhos anteriores (MILES; BIGGS; SCHUBERT, I986; SAUAIA, I997; ARCHER, I990; JACKSON, I995; FERRÁNDEZ; SARRAMNA; TARIN, I977). Desta forma, o questionário continha as seguintes dimensões:

- Auto-avaliação da experiência dos participantes (MILES; BIGGS; SCHUBERT, 1986). Para referendar esta dimensão, perguntou-se em questão aberta, os pontos fortes e fracos percebidos pelos alunos quanto a experiência da participação no Jogo, e também, quanto a operacionalização do Jogo.

- $\quad$ Participação anterior do estudante em Jogos de Empresas (MILEs; BIGGs; SCHUBERT, 1986).

- Estímulos e motivos que contribuíram para o aproveitamento (ARCHER, 1990). Nesta questão questionou-se em pergunta aberta qual foi o estímulo que contribuiu para o aproveitamento, em caso positivo, ou que não contribuiu, para casos em que o estudante não tenha sentido motivação. 
- Benefícios educacionais resultantes, como conhecimentos, habilidades e atitudes (FERRÁNDEZ; SARRAMNA; TARIN, I977).

- $\quad$-Compreensão das regras operacionais do Jogo (JACKSON, I995).

Os dados extraídos dos questionários respondidos pelos alunos foram analisados com base na estatística descritiva, focando a exposição das opiniões dos participantes das simulações realizadas. 


\section{PROCESSAMENTO DOS DADOS E ANÁLISE DOS RESULTADOS}

Com base nas informações e na situação operacional da empresa apresentada, as equipes tiveram que analisá-las e então tomar as decisões. Também cabe lembrar que os testes, questionários e o professor que ministrou a disciplina foram iguais para as duas turmas.

\section{NOTAS DOS TESTES}

Tomando-se por base as notas dos alunos nos pré-teste e pós-teste, conseguidos pelas duas turmas, pode-se afirmar, no teste " $t$ " para dados pareados, considerando o nível de confiança de $99 \%$, ou uma chance de erro de I\%, e uma estatística " $t$ " observada de 3,89, comparada a estatística " $t$ " tabelada de 2,65, percebe-se que o valor encontrado encontra-se na área de rejeição da hipótese nula (Ho). Outra forma de explicar a rejeição de Ho é por meio do nível de significância observado no teste $(\mathrm{P})$ que é menor que I\%, confirmando os achados do estudo (Tabela I), assim pode-se indicar que:

- Rejeita-se $\mathrm{H}_{\mathrm{o}}$, detectando, então que houve um aumento real do desempenho no teste de conhecimentos teóricos após a aplicação do Jogo.

Tabela I Teste T do pré-teste e pós-teste

\begin{tabular}{|lll|}
\hline \multicolumn{2}{|c|}{ Teste $T$ para dados pareados } & Variável 2 (depois) \\
\hline Média & $\mathbf{7 , 1 0}$ & $\mathbf{7 , 6 4}$ \\
\hline Variánel $\mathbf{1}$ (antes) & $\mathbf{1 , 0 4}$ \\
\hline Observações & $\mathbf{1 , 1 0}$ & 62 \\
\hline Stat t & 62 & \\
\hline$P(T<=t)$ & $\mathbf{3 , 8 9}$ & \\
\hline
\end{tabular}

Se admitido que não houve qualquer outro fator, além da vivência no Jogo, atuando de forma sistemática entre as duas mensurações, pode-se concluir que a participação no Jogo pode influenciar de forma contributiva/aditiva no conhecimento teórico em Administração da Produção. 


\section{DESEMPENHO DAS EQUIPES NO JOGO}

O desempenho das equipes no Jogo foi analisado para verificar a existência da relação entre o desempenho das equipes no Jogo (x) com o aumento ou redução da nota no pós-teste quando comparada com o pré-teste (y).

Para mensurar a existência de correlação entre estas duas variáveis $(\mathrm{x}, \mathrm{y})$ da população, utilizou-se do teste de significância $r_{s}$ de Spearman. O coeficiente de correlação de Spearman é definido na equação r:

(I) $\mathrm{r}_{\mathrm{s}}=\mathrm{I}-\underline{\underline{6 . \Sigma \mathrm{D}^{2}}}$

$$
\text { n. }\left(\mathrm{n}^{2}-\mathrm{I}\right)
$$

Onde $\Sigma \mathrm{D}^{2}$ é a diferença quadrática entre os postos.

Tabela 2 Correlação de Spearman

\section{Teste de Significância sobre rs}

\begin{tabular}{|lll|}
\hline & Diurno & Noturno \\
\hline Observações & 12(equipes) & 9 (equipes) \\
\hline rs Spearman & 0,19 & 0,06 \\
\hline
\end{tabular}

Segundo Barbetta (2007), o valor do coeficiente estará no intervalo entre -I a I. Será tão mais próximo de I (ou -I) quanto mais forte for a correlação nos dados observados. Ter-se-á $r_{s}=+\mathrm{I}$ se houver uma correlação positiva perfeita. Por outro lado, ter-se-á $r_{s}=$-I se houver uma correlação negativa perfeita. Em outras palavras, o coeficiente de correlação tenta verificar o alinhamento entre duas variáveis.

Desta forma, no caso da turma do período diurno, $\operatorname{com} \mathrm{n}=\mathrm{I} 2$, valor $r_{\text {sdiurno }}$ $=0,19$ (Tabela 2) e nível de significância de $5 \%$, é possível afirmar que o teste não acusa significância. Não é possível dizer que existe correlação entre desempenho no Jogo e a média da variação da nota nos testes das equipes.

Também, o teste com a turma do período noturno apontou, $\operatorname{com} \mathrm{n}=9$, valor $r_{\text {snoturno }}=0,06$ (Tabela 2) para a não rejeição de $\mathrm{H}_{0}$

- Não rejeita-se $\mathrm{H}_{\mathrm{o}}$, ou seja, as variáveis x e y são não-correlacionadas.

Portanto, a correlação positiva fraca $\left(r_{\text {sdiurno }}=0,19 \mathrm{e} r_{\text {snoturno }}=0,06\right)$, descrita pelos dados, não é suficiente para afirmar a existência de correlação entre as duas variáveis. 
Com estes dados pode-se inferir que o bom desempenho alcançado no Jogo não é o único fator que pode gerar melhorias no conhecimento específico em Administração da Produção, ou seja, o aluno que errou durante a execução do Jogo pode ter tido um melhor aprendizado do que aquele que teve mais acertos nas decisões relacionadas ao Jogo. $\mathrm{Na}$ própria pedagogia atual é discutida a importância do erro no processo de aprendizagem (HOFFMAN, 1997), pois muitas vezes o aluno aprende mais nele do que nos momentos em que está acertando. O erro quando percebido pelo aluno, ou apontado pelo professor, tende a desencadear um processo de reflexão, levando o aluno a verificar porque errou e o que deveria ter feito. Refletindo sobre suas atividades e atitudes o aluno que errou consegue identificar novas situações e possibilidades que o aluno que "acertou" não irá descobrir, ou "aprender". O acerto não gera tal processo de reflexão, e deste modo não pode ser considerado que o aluno que errou não aprendeu o que o professor almejava com a atividade.

Além disso, de acordo com Stahl e Lopes (2004), os modelos dos jogos de empresas, tradicionalmente, apoiam-se no desempenho da empresa fictícia na simulação para avaliar o nível de aprendizagem obtido pelos participantes. Ao observar os resultados obtidos, portanto, pode-se considerar que o uso exclusivo do desempenho no Jogo não é suficiente para verificar o aprendizado aluno. Corroborando com Stahl e Lopes (2004) sugeremse alternativas para uma avaliação qualitativa que possibilite verificar o aprendizado no que diz respeito aos conhecimentos teóricos, habilidades e atitudes, valorizando ainda mais o uso de jogos na formação profissional.

\section{QUESTIONÁRIO DE AVALIAÇÃO}

Após a aplicação do Jogo e dos testes pediu-se aos alunos para que respondessem um questionário estruturado com três (3) perguntas fechadas e uma (I) pergunta aberta.

A primeira pergunta tratou de questionar a experiência dos estudantes em participar de outros Jogos Empresariais. No Gráfico r é possível observar que $68 \%$ dos alunos já tiveram da vivência com outros Jogos Empresariais e $32 \%$ nunca haviam participado de Jogos. 
Gráfico I Participação dos alunos em Jogos Empresariais

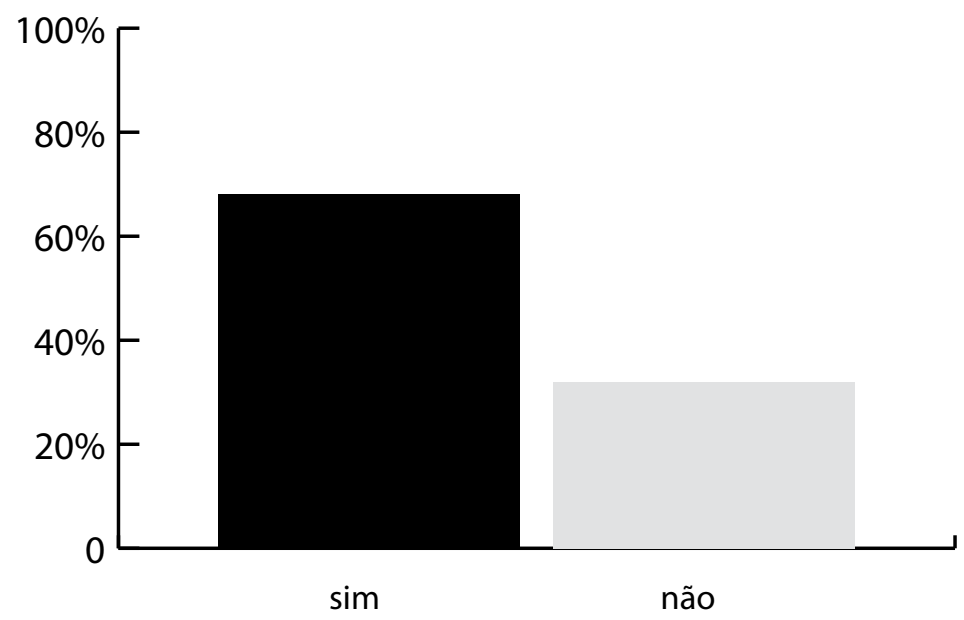

Este resultado mostra a oportunidade que se tem para trabalhar e pesquisar novas estratégias de aprendizado no âmbito geral do curso de Administração, da Universidade Autônoma de Lisboa, visto que os alunos participantes são alunos formandos no curso, e, portanto, não terão oportunidade de vivenciar o uso de Jogos Empresariais novamente durante o curso em questão.

Com relação a intensidade percebida quanto aos benefícios educacionais alcançados na vivência como, conhecimentos, habilidades e atitudes (FERRÁNDEZ; SARRAMNA; TARIN, I977), 3\% disseram ser muito alta, 33\% alta, $58 \%$ razoável, e 5\% baixa (Gráfico 2 ).

Percebe-se que o Jogo foi percebido por grande parte dos participantes como um meio para acessar conhecimentos, habilidades e atitudes, ou seja, mais do que simplesmente um método lúdico, o Jogo foi capaz de proporcionar aprendizagem. Entretanto, o elevado número da opção razoável (58\%) aponta para inconsistências no Jogo quanto aos benefícios educacionais. Ao se analisar os pontos fracos da vivência no Jogo vários alunos citaram a curta duração do Jogo e a falta de outras rodadas de aplicação. Estas informações podem levar ao entendimento de que se houvessem mais rodadas os benefícios educacionais seriam mais evidentes. 
Gráfico 2 Intensidade percebida quanto aos benefícios educacionais alcançados

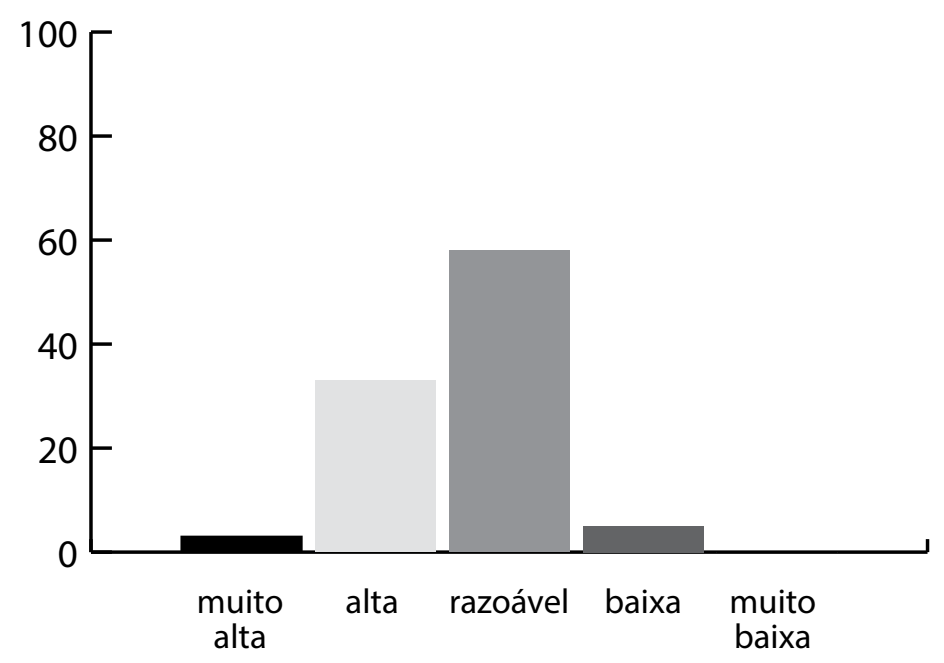

Quanto à dimensão da compreensão das regras operacionais doJogo (JACKSON, 1995), 63\% disseram haver compreendido, pelo menos razoavelmente as regras, enquanto $38 \%$ disseram haver compreendido as regras do Jogo (Gráfico 3).

Gráfico 3 Compreensão das regras do jogo

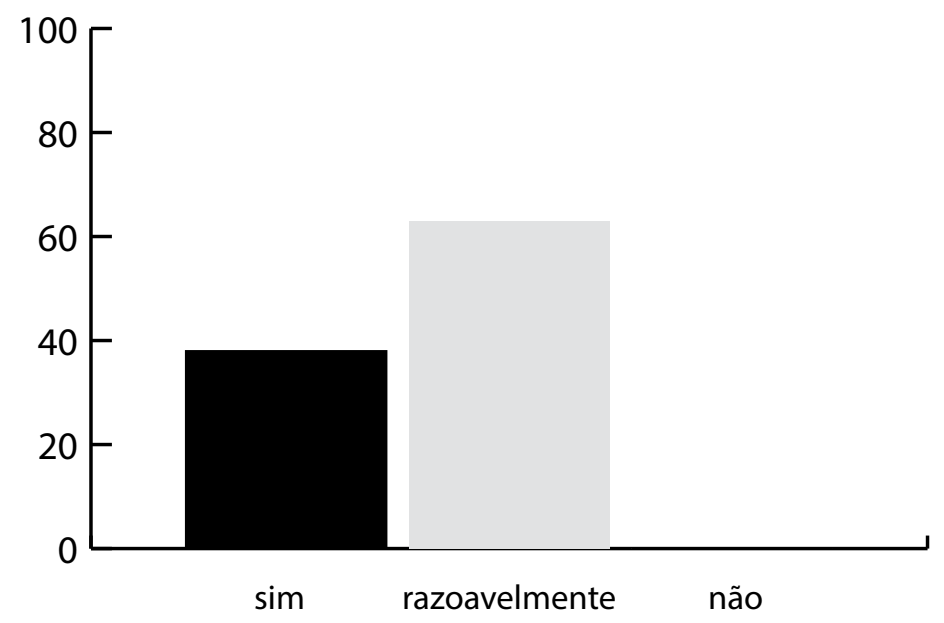


O alto número de alunos (63\%) terem compreendido as regras Jogo razoavelmente aponta para inconsistências na explanação das regras, no Manual do Jogador ou na própria construção do Jogo. Este é um ponto de destaque para reconsiderações a fazer no modelo de Jogo proposto.

Por fim, na quarta pergunta, relacionada aos pontos fracos e fortes da experiência dos participantes no jogo (MILES; BIGGS; SCHUBERT, I986), e da operacionalização do Jogo, obteve-se, principalmente, as seguintes respostas (Quadros 4 e 5).

Quadro 4 Principais respostas quanto aos pontos fracos do Jogo

\begin{tabular}{|c|}
\hline -“Discordância no grupo”. \\
\hline -“Curta duração". \\
\hline $\begin{array}{l}\text {-"Muito tempo despendido num final de curso, o que se torna um problema serio, visto que pode } \\
\text { gerar ineficácia”. }\end{array}$ \\
\hline -“Não dar grande importância as análises". \\
\hline -"Informação reduzida apesar de ser um jogo onde conta o conhecimento e a capacidade de interpretar". \\
\hline -“Não fazer a segunda jogada”. \\
\hline -"Distribuição do capital não ter sido fácil pelos vários fatores da empresa”. \\
\hline -"Muitas opções similares, torna-se cansativo para a leitura e análise”. \\
\hline -"Falta de experiência neste tipo de jogo". \\
\hline -“Falta de experiência em relação a investimentos". \\
\hline $\begin{array}{l}\text {-“O jogo devia ser em formato informático e devia ter mais do que uma jogada, de modo a poder } \\
\text { corrigir alguns pontos”. }\end{array}$ \\
\hline -"Verdadeiro conhecimento do funcionamento da empresa". \\
\hline -“Não colocar mais aprofundamento do funcionamento e do problema de pesquisa”. \\
\hline -"Demasiado texto". \\
\hline
\end{tabular}

Quadro 5 Principais respostas quanto aos pontos fortes do Jogo

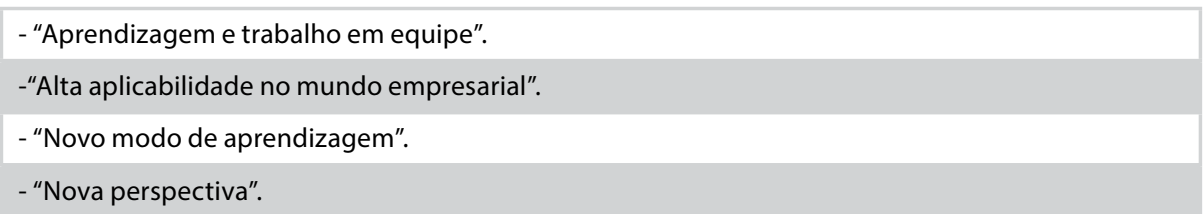


- "A interação entre os elementos do grupo e a noção de que existem algumas diferenças de opinião contribuem favoravelmente para melhores resultados".

- "Melhora o nosso conhecimento, por em prática os conhecimentos adquiridos e melhorar a nossa aprendizagem em relacionamento de grupo".

- “Deu-me uma noção das muitas perspectivas que são necessárias observar na empresa, como elas interagem e quais suas relevâncias na empresa".

- "Realça a intuição de que estamos verdadeiramente numa empresa e aumenta as nossas capacidades".

-“Conseguimos perceber os vários drives fundamentais num processo de produção”.

-"Tentar perceber a dinâmica do jogo, leitura do manual RCAP para melhor entendermos os fatores de prática e resultados".

-"Conhecimento que se adquiriu".

-"Ser uma ideia inovadora, que nos deu uma ideia de como funcionava na prática e ter uma ideia da realidade, e como se combina as assertivas".

-“Fácil compreensão".

-"Este jogo mostra as dificuldades que podemos encontrar".

-“Como era a primeira vez que jogava foi positivo para a minha experiência".

Para um melhor entendimento as respostas foram agrupadas em fatores de acordo com similaridades (Quadro 6).

Quadro 6 Pontos fortes e fracos do Jogo

\begin{tabular}{|ll|}
\hline Pontos Fortes & Pontos Fracos \\
\hline Experiência prática & Design do simulador \\
\hline Ampliação do conhecimento & Forma de aplicação \\
\hline Aprendizagem de forma inovadora & Falta de conhecimento prévio no assunto \\
\hline Aproximação com a realidade & \\
\hline Trabalho em equipe & \\
\hline
\end{tabular}

Quanto aos pontos fracos (Quadros 4 e 6) relacionados ao "Design do simulador" os principais apontamentos estiveram em torno do número excessivo de assertivas componentes do Simulador e a falta de interatividade do mesmo.

Já, quanto aos pontos fracos relacionados a "Forma de aplicação", o principal argumento estava relacionado ao pouco tempo para tomada 
de decisões, já que o Jogo exigia muita leitura, e poucas rodadas. Neste sentido, Bernard (2006) menciona que o tempo disponível para a aplicação de simuladores pode ser um fator limitante. Esta limitação será tão maior quanto mais complexo for o simulador a ser utilizado. Portanto, se o professor dispõe de apenas oito horas para realizar uma simulação, a maior parte dos simuladores do tipo genérico, mais abrangentes, não poderá ser utilizada, porque demanda mais tempo do que o disponível para o seu entendimento e utilização de rodadas consideradas satisfatórias. Apesar do simulador aqui proposto não ser considerado genérico e abrangente para todas as áreas da empresa, ou seja, um simulador específico de Administração da Produção, a forma de aplicação foi considerada um ponto fraco.

Em relação aos pontos fortes (Quadros 5 e 6), houve ênfase na experiência prática e aproximação com a realidade. Quanto a esse ponto, é importante destacar que esse resultado difere dos resultados alcançados por Johnsson (2006), onde os participantes quase não consideraram a experiência prática proporcionada pelo Jogo como ponto que provocasse um maior desenvolvimento das habilidades gerenciais. Como os participantes da pesquisa de Johnsson (2006) eram estudantes de pós-graduação, é possível que quanto maior o nível instrucional menor é a percepção quanto aos benefícios da experiência prática na vivência de um Jogo Empresarial. O mesmo argumento pode ser mencionado com relação ao ponto forte aproximação com a realidade, onde no trabalho de Johnsson (2006), também, poucos participantes consideraram a aproximação com a realidade um ponto forte da vivência. Estes resultados monstram que o Jogo pode desenvolvido pode ser utilizado como um modo de integração entre teoria e prática, como sugere Sauaia (1995), Rosas (2004) e Tanabe (1977).

Portanto, a avaliação fundamentou-se em três pilares. Inicialmente aplicou-se o pré-teste, em que os alunos foram solicitados a responder questões teóricas que seriam exigidas durante o jogo e ao final, o pós-teste, para aferição do crescimento do seu conhecimento. A segunda etapa da avaliação levou em consideração o desempenho das equipes no jogo e o espectro de escores obtidos. Finalmente, solicitou-se o preenchimento de um questionário de auto-avaliação, que permitiu o levantamento da opinião 
do aluno sobre a aceitação e sentimento de utilidade da proposta para a sua formação.

Quanto à primeira etapa descrita e aplicando-se o teste " $\mathrm{t}$ " de student, pode-se constatar que há uma relação entre o aumento do conhecimento dos alunos e a aplicação do jogo. Em outras palavras, a prática do jogo levou ao incremento da base teórica dos alunos.

A segunda etapa nos mostra que o aumento da nota do pré para o pósteste não está relacionada com o desempenho obtido no jogo. Isto quer dizer que jogar é positivo e apoia a aprendizagem; porém, vencer não é fator relevante se correlacionado à aprendizagem.

A terceira etapa da avaliação aponta para várias constatações importantes. Infere que decisões coletivas são melhores que as individuais; que se exercita o trabalho em equipe; que este método introduz uma nova forma de aprendizagem, com algum componente lúdico; que o modelo reproduz o ambiente empresarial. Em síntese, o jogo proposto é útil pelos benefícios percebidos, inovador pelos desafios apresentados (em especial pela base teórica que o suporta) e estimulante, pela aproximação com os desafios da realidade de um administrador. 


\section{CONSIDERAÇÕES FINAIS}

Dos resultados apresentados pode-se concluir, quanto à avaliação do comportamento e aprendizagem dos participantes e da técnica de ensino, que o Jogo se mostrou positivo. Por meio do teste " $\mathrm{t}$ " de student aplicado as notas do pré e pós-teste, pode-se inferir que há uma relação entre o aumento do conhecimento dos alunos e a aplicação do jogo. Em outras palavras, a prática do jogo levou ao incremento da base teórica dos alunos. Já quando a média das variações das notas das equipes foi comparada ao desempenho no Jogo, observou-se que este aumento da nota do pré para o pós-teste não estava relacionada com o desempenho obtido no jogo. Isto quer dizer que jogar é positivo e apoia a aprendizagem; porém, vencer não é fator relevante se correlacionado à aprendizagem.

Ainda, por meio do questionário de auto-avaliação foi possível concluir, por meio da explanação dos alunos, que o jogo mostrou-se motivador e estimulante, pela aproximação com os desafios da realidade de um administrador e pela inovação no processo de aprendizagem. Além disso, os alunos apontaram como benefícios do trabalho em equipe e da aprendizagem de conhecimentos teóricos baseados em uma perspectiva diferenciada.

Quanto ao funcionamento do Jogo deve-se levar em consideração alguns aspectos citados como pontos fracos no questionário de auto-avaliação. Incluem-se aplicação de curta duração, demasiado texto que tornou cansativo a análise e leitura do Jogo, bem como, a interface do modelo.

Estes resultados, positivos quanto à avaliação do comportamento e aprendizagem dos participantes e da técnica de ensino, e com algumas ressalvas quanto ao funcionamento do Jogo, indicam a necessidade de continuar a busca do desenvolvimento e aperfeiçoamento deste novo modelo de Jogo Empresarial a fim de desenvolver uma ferramenta que contribua no processo de ensino-aprendizagem em Administração.

Com relação às limitações do trabalho, o fato dos autores serem os professores que aplicaram o teste e o jogo pode gerar vieses quanto às conclusões do trabalho. Em que pese os resultados apresentarem-se positivos 
em relação aos pressupostos dos autores, deve-se considerar o fato de que os pesquisadores, com seu entusiasmo, terem influenciado a obtenção dos resultados na direção dos seus pressupostos.

Deve-se considerar também que a diferença entre quaisquer grupos deriva de vários aspectos, que não só a capacidade ou o processo de aprendizagem intrínseco; depende de questões atitudinais, de eventuais fontes de dispersão de interesses, do momento vivido.

Além disso, para o escopo deste artigo não foi possível avaliar a diferença entre grupos com maior ou menor desempenho no jogo e nos testes com as atitudes dos participantes, entretanto, sugere-se que outros estudos avancem neste quesito.

Também, como proposta para novos estudos sugere-se o uso de outras técnicas de análise, como por exemplo, técnicas multivariadas que combinam a análise dos dados levantados no estudo. Além disso, possíveis novos estudos podem ser direcionados para situações com maior domínio dos diversos aspectos aqui mencionados. Desta maneira poder-se-ia ter maior clareza sobre os resultados que são objetos desta análise. Além disso, recomenda-se novos estudos com mais evidências empíricas sobre a relação entre o aprendizado e o uso de Jogos de Empresas, bem como, sobre as vantagens e desvantagens do uso da técnica de Jogos de Empresas das técnicas tradicionais de ensino e aprendizagem, pois até o momento, poucos são os estudos que tem evidenciado essa dinâmica, e estes aparecem até o momento inconclusivos. 


\section{REFERENNCIAS}

ANDRADE, R. O. B; SCAICO, O.; FERRAZ, S. F.; SALEM, I. M. M. Pesquisa nacional sobre o perfil, formação, atuação e oportunidades de trabalho do administrador. Brasília: CFA, 2004.

ANDRADE, M. M. Como preparar trabalhos para cursos de pós-graduação: noções práticas. 5.ed. São Paulo: Atlas, 2002.

ARCHER, E. R.. O mito da motivação. In: BERGAMINI, C. W.; CODA, R. Psicodinâmica da vida organizacional. São Paulo: Pioneira, 1990, p. 3-24.

BARBETTA, P. B. Estatística aplicada às ciências sociais. Florianópolis: UFSC, 2007.

BADURDEEN, F.; MARKSBERRY, P.; HALL, A.; GREGORY, B. Teaching Lean Manufacturing With Simulations and Games: A Survey and Future Directions. Simulation \& Gaming, v.41, n.4, p. 465-486, 2010.

BERNARD, R. R. S. Estrutura de utilização dos jogos de empresas nos cursos de graduação em administração e ciências contábeis do país e avaliações preliminares de uma disciplina baseada neste método. In: Enangrad, 17., 2006, São Luiz. Anais... São Luiz: Angrad, 2006.

BOUYER, G.C.; SZNELWAR, L.; COSTA, M.J.B. Aprendizagem estratégica e aquisição conceitual no ensino de gestão de operações. In: Encontro Nacional De Engenharia De Produção, 25., 2005, Porto Alegre. Anais... Porto Alegre, 2005.

CARNIEL, A. Especificação de um ambiente para o trabalho e o aprendizado em grupos colaborativos na Internet. Dissertação (Mestrado em Engenharia de Produção e Sistemas). UFSC, Florianópolis, 2002.

ESCRIVÃO FILHO, E.; RIBEIRO, L. R. Inovando no Ensino de Administração: Uma Experiência com a Aprendizagem Baseada em Problemas (PBL). In: Enepq, 1., 2007, Recife/PE. Anais... Recife/PE: Anpad, 2007.

FARIA, A. A. O Uso Educacional dos Computadores: Um Estudo da Formação dos Administradores de Empresas. Dissertação (Mestrado em Administração de Empresas). EAESP-FGV, São Paulo, 1997.

FERRANDÉZ, A.; SARRAMONA, J.; TARIN, L. Tecnología didáctica: teoría y práctica de la programación escolar. Barcelona, CEAC, 1977.

FOX, S. From management education and development to the study of management learning. In: BURGOYNE, J.; REYNOLDS, M. Management learning: integrating perspectives in theory and practice. London: Sage Publications, 1997. p. 21-37.

GERBER, J. Z. Proposta de Metodologia para o Desenvolvimento de Recursos à Aplicação de Jogos de Empresas via Internet. Dissertação (Mestrado em Engenharia de Produção e Sistemas). UFSC, Florianópolis, 2000.

GOMES, G.; TORRENS, E. W.; CUNHA, P. R. Motivação e Resistência ao Uso da Tecnologia da Informação: Um Estudo Entre Professores. Administração: Ensino e Pesquisa, v. 13, n. 2, p. 301-324, 2012. 
GRAMIGNA, M. R. M.. Jogos de empresas e técnicas vivenciais. São Paulo: Makron Books, 1995.

HAIDT, R. C. C. Curso de didática geral. São Paulo: Ática, 1995.

JARVIS, P. Adult and Continuing Education: Theory and Practice. New York: Routledge, 1995.

JACKSON, T. European management learning: a cross-cultural interpretation of Kolb's learning cycle. Journal of Management Development. England, MCB University Press, v.14, n.6, p.42-50, 1995.

JÚNIOR, N. T.; SOUZA, G. G.; NASCIMENTO, J. Z. Análise de processos por meio da simulação computacional: proposta de uma estratégia de ensino na graduação em Administração. Administração: Ensino e Pesquisa, v. 13, n. 3, p. 491-522, 2012.

KOLB, D. A.; RUBIN, I. M.; McINTYRE, J. M. Psicologia Organizacional: uma abordagem vivencial. São Paulo: Atlas, 1978.

LACRUZ, A.J. Jogos de empresas: considerações teóricas. Caderno de Pesquisas em Administração, v.11, n.4, p.93-109, 2004.

MAÑAS, A. V. O processo do ensino em administração: a relação qualidade, produtividade e imagem - um modelo de avaliação. In: Enangrad, 7., 1996, Florianópolis. Anais... Florianópolis: Angrad, 1996.

MARTINELLI, D. P. A utilização dos jogos de empresas no ensino de administração. Dissertação (Mestrado em Economia, Administração e Contabilidade). FEA-USP, São Paulo, 1987.

MILES, W. G.; BIGGS, W. D.; SCHUBERT, J. N. Student perceptions of skill acquisition through cases and general management games: a comparison. Simulation \& Games, v. 17, n. 1, p. 7-24, 1986.

MINTZBERG, H. MBA? Não obrigado: uma visão crítica sobre a gestão e o desenvolvimento de gerentes. Porto Alegre: Bookman, 2006.

MOSCOVICI, F. Desenvolvimento interpessoal. 3.ed. Rio de Janeiro: Livros Técnicos e Científicos, 1985.

MOTTA, G. S.; MELO, D. R. A.; PAIXÃO, R. B. O jogo de empresas no processo de aprendizagem em administração: o discurso coletivo de alunos. Revista de Administração Contemporânea, v. 16, n. 3, p. 343-359, 2012.

OLIVEIRA, M. A. Implantando o Laboratório de Gestão: um programa integrado de educação gerencial e pesquisa em administração. Tese (Doutorado em Administração, Economia e Contabilidade). FEA-USP, São Paulo, 2009.

OLIVEIRA, M. A.; SAUAIA, A. C. A. Impressão Docente para Aprendizagem Vivencial: um Estudo dos Benefícios dos Jogos de Empresas. Administração: Ensino e Pesquisa, v. 12, n. 3, p. 355-391, 2011. 
OLIVIER, M.; ROSAS, A. R.. Jogos de empresas na graduação e no mestrado. In: Seminários em Administração FEA-USP, 4., 2004, São Paulo. Anais...São Paulo: FEA/ USP, 2004.

PROTIL, R. M. Utilização de simuladores empresariais no ensino de ciências sociais aplicadas: um estudo na República Federal da Alemanha. Revista de Economia, v.31, n.2, p. 113-134, 2005.

SAUAIA, A. C. A. Satisfação e aprendizagem em jogos de empresas: contribuições para a educação gerencial. 273f. Tese (Doutorado em Administração). FEA-USP, São Paulo, 1995.

SAUAIA, A. C. A. Jogos de empresas: aprendizagem com satisfação. Revista de Administração, v.32, n.3, p. 13-27, 1997.

SILVA, C. R. O. Bases Pedagógicas e Ergonômicas para a Concepção e Avaliação de Produtos Educacionais Informatizados. Dissertação (Mestrado em Engenharia de Produção).

UFSC, Florianópolis, 1998.

SILVA, A. D. Administração Da Produção - Mudanças Na Prática De Ensino. Administração: Ensino e Pesquisa (artigo de divulgação). Disponível em: http://www.angrad.org.br/_resources/files/_modules/producao/ producao_419_201212051834228e9c.pdf. Acesso em: 14/09/2012.

STAHL, L. M.; LOPES, P. C. Estratégias de Avaliação para Jogos de Empresas Gerais: Avaliando Desempenho ou Aprendizagem. In: EnANPAD, 28., 2004, Curitiba. Anais... Curitiba: ANPAD, 2004.

TANABE, M. Jogos de Empresas. Dissertação (Mestrado em Economia) FEA-USP, São Paulo, 1977.

TAPSCOTT, D. Growing Up Digital: The Rise of the Net Generation. McGraw Hill, New York, 1997.

TEACH, R. Profits: the false prophet. Development In Business Simulation \& Experiential Exercises, v. 14, n. 1, p. 205-207, 1987. 


\section{DADOS DOS AUTORES}

Janaina Piana janainapiana@gmail.com

Mestre em Administração pela UFSC

Instituição de vinculação: Fundação Getúlio Vargas - Escola Brasileira de Administração Pública e de Empresas

Rio de Janeiro/RJ - Brasil

Áreas de interesse em pesquisa: Gestão da Inovação e Administração da Produção.

* Rua Marques de Abrantes, 177 Flamengo Rio de Janeiro/RJ 22230-060

ROLF HERMANN ERDMANN rolf.erdmann@ufsc.br

Doutor em Administração pela UFSC

Instituição de vinculação atual: Universidade Federal de Santa Catarina

Florianópolis/SC - Brasil

Áreas de interesse em pesquisa: Administração da Produção e Desempenho da Gestão das Operações. 